\title{
Effects of $n-3$ Supplementation on Plasma and Liver Phospholipid Fatty Acids Profile in Aged Wistar Rats
}

\author{
Tamara Popović, ${ }^{\mathrm{a}, *}$ Sunčica Borozan,${ }^{\mathrm{c}}$ Aleksandra Arsić, ${ }^{\mathrm{a}}$ Jasmina Debeljak-Martačić, ${ }^{\mathrm{a}}$ \\ Vesna Vučić, ${ }^{\mathrm{a}}$ Silvio de Luka, ${ }^{\mathrm{b}}$ Ivan Milovanović, ${ }^{\mathrm{b}}$ Aleksandar Trbović, ${ }^{\mathrm{a}}$ and Maria Glibetić ${ }^{\mathrm{a}}$ \\ anstitute for Medical Research, University of Belgrade, Tadeusa Koscuska 1,11000 Belgrade,Serbia \\ ${ }^{\mathrm{b}}$ Institute for pathological physiology, Faculty of Medicine, Dr. Subotića 8,11000 Belgrade,Serbia \\ ${ }^{\mathrm{c}}$ Faculty of Veterinary Medicine, University of Belgrade, Bulevar Oslobodjenja 18,11000 Belgrade,Serbia
}

RECEIVED SEPTEMBER 10, 2010; REVISED FEBRUARY 2, 2011; ACCEPTED FEBRUARY 11, 2011

\begin{abstract}
The effects of fish oil supplementation in Wistar rats are focused on cardiovascular, endocrine, metabolic and antioxidant status changes. We determined plasma and liver phospholipid fatty acids (FAs) status and plasma lipid concentrations in aged Wistar rats. Our results showed differences in plasma and liver FAs profiles as well as plasma chlolesterol (CHOL), triglicerides (TG), high density lipoproteins (HDL), low density lipoproteins (LDL), CHOL/HDL ratio (risk factor for atherosclerosis) and LDL/HDL ratio (risk for cardiovascular diseases) between treated and control group of animals. In fish oil treated group there were statistically significant changes in FAs profile in increasing linoleic acid (LA), dihomo- $\gamma$ linoleic acid, eicopentanoic acid (EPA) and docosapentaenoic acid (DPA) and decreasing in arachidonic acid (AA) concentration. Also, liver phospholipids FAs results showed increasing concentrations of vascenic acid, LA, EPA, and DPA and decreasing concentration of AA after supplementation of fish oil compared to control group. However, concentrations of CHOL, LDL and non HDL concentrations decreased while HDL increased in fish oil group. CHOL/HDL, LDL/HDL ratios decreased. These findings suggest that long term treatment of fish oil in aged Wistar rats can be beneficial in decreasing LDL, and decreasing risk factors for developing atherosclerosis and cardiovascular diseases. (doi: 10.5562/cca1751)
\end{abstract}

Keywords: fish oil, aged rats, fatty acids, lipid status

\section{INTRODUCTION}

The phospholipid class, FAs composition and cholesterol content in biomembranes are basic determinants of the physical properties of membranes. They have been shown to influence a wide variety of membranedependent functions such as membrane transport, enzyme activity and receptor function. ${ }^{1}$ Biological membranes are organized assemblies of lipids and proteins with small amount of carbohydrate. Membrane proteins carry out the dinamic processes associated with membrane. ${ }^{2}$ In higher plants and animals the predominant FAs residues are those of the $\mathrm{C} 16$ and $\mathrm{C} 18$ species palmitic, linoleic and stearic acids. Over one half of the FAs residues are unsaturated and are often polyunsaturated. ${ }^{3}$ Plasma lipoproteins are clasified into five categories: chylomicrons (transport exogenous TG and CHOL from the intestine to the tissues), VLDL, IDL, LDL (transport endogenous TG and CHOL from the liver to the tissues) and HDL (transport endogenous CHOL from the tissus to the liver). ${ }^{4}$
Changes in phospholipid structure contents is a major marker of atherosclerotic changes. ${ }^{5}$ Greater intake of the long-chain $n-3$ FAs, EPA and docosahexaenoic acid (DHA) has been recommended by a variety of scientific and regulatory bodies to reduce population risk for coronary artery disease (CAD) ${ }^{6}$

The FAs profile of the serum phospholipid is related to the average dietary FAs intake during the last 3 to 6 weeks, while the composition of erythrocyte phospholipids depends on the dietary fat intake during the preceding months. ${ }^{7}$ The FAs profile in tissues partly reflects not only the dietary fat intake, but also the efficiency of FAs metabolism in the body. ${ }^{8}$

Although mammals are able to synthesize saturated FAs from nonfat precursors and unsaturated fatty acids of the $n-9$ and $n-7$ series, they lack the $\Delta 12$ and $\Delta 15$ desaturase enzymes (found in most plants) for insertion of a double bond at the $n-6$ or $n-3$ position. Thus mammalian cells can not synthesize $n-6$ or $n-3$ polyunsaturated FAs (PUFAs) de novo. The commonly consumed PUFAs are linoleic acid (LA) $(18: 2, n-6)$ and

\footnotetext{
* Author to whom correspondence should be addressed. (E-mail: poptam@gmail.com)
} 
$\alpha$-linolenic acid (ALA) (18:3, n-3). Once consumed these FAs can be converted to longer chain, more unsaturated derivatives. ${ }^{9}$ LA is converted via $\gamma$-linolenic $(18: 3, n-6)$ and dihomo- $\gamma$-linolenic $(20: 3, n-6)$ acids to arachidonic acid (AA; 20:4, $n-6$ ). ALA is converted to EPA $(20: 5, n-3)$ and DPA $(22: 5, n-3)$. EPA and DHA are termed long chain $n-3$ PUFAs. These FAs are found in oily fish and the preparations known as fish oil. ${ }^{9}$ They are involved in regulation of lipid metabolism and processes involved in lipid transport and targeting to tissues. ${ }^{9}$ They are components of membranes of all cells, contributing to the fluidity of the membrane and this is believed to play a role in regulation of activity of membrane proteins. Also PUFAs are substrates for the synthesis of bioactive molecules such as prostaglandins (PG), thromboxanes (TX) and leukotrienes (LT).10 Eicosanoids formed from the $n-3$ FA are much less potent in causing biological responses, including stimulation of cytokine production and inflammatory reactions than those formed from the $n-6$ series. ${ }^{10}$ The immune and inflammatory cells contain many transcription factors including NF kappa B (NFkB) which regulates the synthesis of cytokines interleukin 1 (IL-1), interleukin 2 (IL2), interleukin 6 (IL-6), tumor necrosis factor$\alpha(\mathrm{TNF}-\alpha)$ and interferon- $\beta$. EPA reduces the production of pro-inflammatory IL- 1 and IL- 6 as well as TNF- $\alpha$ and $-\beta$ in response to an inflammatory stimulus. ${ }^{10}$

When fish oil is provided EPA is incorporated into cell membrane phospholipids, partly at the expense of AA. ${ }^{9}$ There is less AA available for eicosanoid synthesis. EPA inhibits the oxidation of AA by cyclooxygenase $(\mathrm{COX})$. Fish oil decreases production of prostaglandin (PG) like PGE2, of thromboxane (TX) like TXA2 and leukotrienes (LT) like LTB4.

PUFAs can potentially reduce platelet aggregation, blood clotting, smooth muscle contraction, and leukocyte chemotaxis, and can modulate inflamatory cytokine production and immune function. ${ }^{9}$

Fish oils which are rich in $n-3$ PUFAs have been postulated to be beneficial in several disease states including atherosclerosis, hypertension and arthritis. ${ }^{11,12}$ The major $n-3$ FAs found in fish oil are EPA [20:5 $(n-3)]$ and DHA $(22: 6, n-3)$. Fish oils have been shown to reduce hepatic lipogenesis and VLDL secretion and increase post-heparin plasma LPL activity. ${ }^{13}$ Fish oil rich in EPA and DHA inhibits production of PGE2, as well as lymphocyte proliferation and production of interferon- $\gamma$ (IFN- $\gamma){ }^{10}$ Immune function could be improved by PUFAs and among them an $n-3$ FAs from fish oil EPA possess the most potent immunomodulatory activities. ${ }^{10}$

An increasing number of clinical studies indicate that the consumption of ALA has beneficial effects on human health, particularly on cardiovascular diseases
(CVD). ALA consumption is protective against cardiac death and nonfatal myocardial infarction (MI) in prospective and intervention trials. ${ }^{14}$ The proportion of saturated (SA), monounsaturated (MUFAs), and PUFAs fat content may have an effect not only on the ALA conversion into EPA and DHA, but also on the concentrations of inflammatory markers and on the plasma lipid profile. $^{15,16}$

Liver has important role in synthesis and metabolism of phospholipids. Liver phospholipids build structure of hepatocyte membrane, takes part in metabolic activities and repairing after metabolic disturbances. Some of them lipoproteins are in circulation and a part are excreted in bile. Phospholipids of hepatocytes and plasma has more percentage of stearic and AA. ${ }^{17}$

A large body of evidence shows that hepatic lipogenesis is regulated by both nutritional and hormonal factors. In particular, it is well documented that dietary PUFAs are noticeably effective in inhibiting hepatic lipogenesis and in lowering hypertriglyceridemia, $n-3$ FAs being more potent than $n-6$ lipids in this respect. ${ }^{17}$ The $n-3$ PUFAs also decrease production of AA derived eicosanoids. Due to lack of $\Delta 6$ desaturase, content of AA in inflammatory cells depend on the uptake of AA synthesized in the liver from plasma it depends on hepatic desaturases. ${ }^{10}$

Furthermore, dietary PUFAs coordinately decrease the expression of hepatic genes encoding glycolytic and lipogenic regulatory enzymes involved in the flux of glucose to FAs. ${ }^{18}$ The $n-3$ PUFAs also play a crucial role as "fuel partitioners", in that they direct FAs away from triacylglycerol storage and toward oxidation. ${ }^{19}$ They act by upregulating the expression of genes encoding proteins involved in FAs oxidation while down regulating genes encoding proteins of lipid synthesis. ${ }^{20}$ On the other hand, MUFAs oleate $(\mathrm{C} 18: 1, n-9)$ or SFA like palmitate $(\mathrm{C} 16: 0)$ and medium-chain FAs [as present in coconut oil (CO)] do not inhibit either the activities or the expression of the lipogenic enzymes. ${ }^{21}$

Palmitat (16:0) the normal product of FAs synthesis pathway is the precursor of longer-chain saturated and unsaturated FAs through the actions of elongases and desaturases. Elongases are present in both the mitochondrion and the endoplasmatic reticulum but the mechanisams of elongation at the two sides differ. The difference is in final reduction terminal redox coenzyme. $^{22}$ Unsaturated FAs are produced by terminal desaturases. Mamals systems contain four terminal desaturases of broad chain-lenth specificities designated $\Delta 9, \Delta 6, \Delta 5$, and $\Delta 4$ fatty acyl-CoA desaturases. Mammalian terminal desaturases are components of cytochrome b5 and NADH-cytochrome b5 reductase. A variety of unsaturated FAs may be synthesized by combinations of elongation and desaturation. ${ }^{22}$ 


\section{EXPERIMENTAL}

\section{Materials and Methods}

The experiments were carried out on adult male Wistar rats $(280 \mathrm{~g})$ housed in stainless steel cages with wired floors and with free access to food (Veterinarski zavod, Subotica) and water, in a room under controlled conditions ( $12 \mathrm{~h}$ light/dark cycles, temperature $22 \pm 2{ }^{\circ} \mathrm{C}$ ). Rats were randomly assigned to experimental or a control group. All experiments were done according to our Institutional guidelines for animal research and principals of the European Convention for the Protection of Vertebrate Animals Used for Experimental and Other (Official Daily N. L 358/1-358/6, 18, December 1986).

Wistar rats were divided into two groups of ten animals: control and treated group (fish oil, $45 \mathrm{mg}$ EPA and $30 \mathrm{mg}$ DHA per day, Natura Wealth, Nordvik). The model of rat $n-3$ feeding was the modification of animal feeding protocol described by Tepsic et al. ${ }^{23}$ Individually caged, body weight and age-matched rats were treated as follow: control $(n=10)$ and $n-3$ enriched diet $(n=10)$ for 6 weeks. Each morning rats were individually caged and fed only by one briquette saturated with the exact amount $(200 \mu \mathrm{L})$ of saline (control rats) or $200 \mu \mathrm{L}$ fish oil, (45 mg EPA and $30 \mathrm{mg}$ DHA per day) (Natura Wealth, Nordvik). The same amount of food was return to rat cages 30-40 min after individual feeding treatment. The body weight was measured every day and animals were sacrificed in the morning after 12 $\mathrm{h}$ of fasting.

Blood samples $\left(6-8 \mathrm{~cm}^{3}\right)$ from all rats were obtained via aorta abdominalis puncture and collected in tubes containing sodium citrate $(3.8 \% \mathrm{w} / \mathrm{v})$ as anticoagulant. Immediately after sacrificing, part of the liver was frozen at $-80^{\circ} \mathrm{C}$.

\section{Plasma Biochemical Parameters}

Cholesterol and triglyceride concentrations were measured in plasma using the automated enzymatic methods with cholesterol oxidase, and glycerol oxidase, respectively (EliTech Diagnostic, Sees, France). Serum highdensity lipoprotein (HDL) cholesterol was measured after other classes of lipoproteins had been precipitated with phosphotungstic acid and magnesium chloride (Lopes-Virela et al. 1977). ${ }^{24}$ LDL cholesterol was estimated using Friedewald formula (Friedewald et al.1972). ${ }^{25}$

\section{Total Lipid Extraction}

The method consists of homogenizing the tissue with a 2:1 chloroform/methanol mixture and washing with 5 times smaller volume of water or $\mathrm{NaCl}(0.9 \mathrm{~g}$ in $100 \mathrm{~mL}$ of water). The resulting mixture separates into two phases. The lower phase is the total pure lipid extract. In details, liver tissue ( $1 \mathrm{~g})$ was lyophilized and chloro- form/methanol (2:1) and butylhydroxytoluene (BHT) as antioxidant were mixed. When the mixture is allowed to stand a biphasic system was obtained. After evacuation water was added. After centrifugation upper phase were put away until complete separation of the system. Further evacuation was done with $2: 1$ solvent systems: methanol/benzene, acetone/benzene, and ethanol/ benzene. Adding of chlorophorm and its evacuation and after it adding hexane in sample made it ready for thin liquid chromatography (TLC) (23).

\section{FAs Analysis}

The phospholipid fraction was isolated from the extracted lipids by one-dimensional (TLC) neutral lipid solvent system of hexane:diethyl ether:acetic acid (87:2:1) using Silica Gel GF plates (C. Merck, Darmstadt, Germany). The phospholipid fraction was scraped into glass tubes and phospholipid FAs methyl esters were prepared by transmethylation with sodium hydroxide $\left(2 \mathrm{~mol} \mathrm{dm}^{-3}\right)$ in methanol (heated at $85^{\circ} \mathrm{C}$ for $1 \mathrm{~h})$ and after that sulfuric acid $\left(1 \mathrm{~mol} \mathrm{dm}^{-3}\right)$ in methanol (heated $85{ }^{\circ} \mathrm{C}$ for $2 \mathrm{~h}$ ). After 30 minutes, samples of esters were centrifuged, upper phase were put into tubes and evaporated with tehnical nitrogen. FAs methyl esters derivatives formed from isolated plasma phospholipids fraction were separated by Gas Chromatography (GC) using Shimadzu GC 2014 equipped with a flame ionization detector and DB-23 fused silica gel capillary column. The flame ionization detector was set at 250 ${ }^{\circ} \mathrm{C}$, the injection port at $220{ }^{\circ} \mathrm{C}$, and the oven temperature programmed from 130 to $190{ }^{\circ} \mathrm{C}$ at the heating rate of $3{ }^{\circ} \mathrm{C} / \mathrm{min}^{26}$

Comparing sample peak retention times with authentic standards (Sigma Chemical Company) and/or the (PUFA)-2 standard mixtures (Restec) identified individual FAs methyl esters.

\section{Estimated Activities of Desaturase System}

Several fatty-acid indexes, reflecting desaturase and elongase activity, were derived from the primary data. ${ }^{27}$ The ratios of $20: 4 / 20: 3,20: 3 / 18: 2$ and 22:6/22:5 were used as a measure of estimated $\Delta 5$-desaturase, $\Delta 6$ desaturase and $\Delta 4$-desaturase activities respectively, while 18:1/18:0 and 18:0/16:0 ratios represented estimated $\Delta 9$-desaturase and elongase activities.

\section{Statistical Analysis}

All the results are expressed as the mean \pm SD. Normality was tested using the Kolmogorov-Smirnov test before statistical analysis. One-way ANOVA and the Student t-test were used to compare the normally distributed variables. Where ANOVA revealed a significant effect, Tukey post hoc test was administered to identify differences between the groups. The differences were considered significant at $p \leq 0.05$. 
Table 1. Serum biochemical parameters in control and treated group of aged Wistar rats

\begin{tabular}{lcccc}
\hline & CHOL $(\mathrm{mmol} / \mathrm{L})$ & HDL $(\mathrm{mmol} / \mathrm{L})$ & LDL $(\mathrm{mmol} / \mathrm{L})$ & TG $(\mathrm{mmol} / \mathrm{L})$ \\
\cline { 2 - 5 } Control group & $0.803 \pm 0.22$ & $0.171 \pm 0.04$ & $0.517 \pm 0.19$ & $0.254 \pm 0.06$ \\
Omega 3-start & $0.74 \pm 0.21$ & $0.16 \pm 0.04$ & $0.45 \pm 0.17$ & $0.31 \pm 0.08$ \\
Omega 3-end & $0.54 \pm 0.14^{* \#}$ & $0.23 \pm 0.07^{* * \#}$ & $0.17 \pm 0.13^{* * * \# \# \#}$ & $0.25 \pm 0.06$ \\
\hline
\end{tabular}

$* p<0.05 ; * * p<0.01 ; * * * p<0.001-$ when compared before and after treatment.

${ }^{\#} p<0.05 ;{ }^{\#} p<0.01,{ }^{\#} p<0.001$ - when compared to control group, as determined by ANOVA followed by the Tukey post hoc test.

Table 2. Atherosclerosis and cardiovascular risk factors in control and treated group of aged Wistar rats

\begin{tabular}{|c|c|c|c|c|}
\hline & NON-HDL & $\mathrm{CHOL} / \mathrm{HDL}$ & LDL/HDL & NON HDL/HDL \\
\hline Control group & $0.65 \pm 0.10$ & $4.89 \pm 1.53$ & $2.80 \pm 0.85$ & $3.89 \pm 1.53$ \\
\hline Omega 3-start & $0.60 \pm 0.14$ & $5.73 \pm 1.96$ & $2.81 \pm 0.94$ & $3.39 \pm 1.04$ \\
\hline Omega 3-end & $0.31 \pm 0.13 * * * \# \#$ & $2.31 \pm 0.61 * * * \# \#$ & $0.82 \pm 0.38 * * * \# \#$ & $1.15 \pm 0.48^{* * \# \#}$ \\
\hline
\end{tabular}

$* p<0.05 ; * * p<0.01 ; * * * p<0.001-$ when compared before and after treatment.

$\# p<0.05 ;{ }^{\#} p<0.01,{ }^{\#} p<0.001$ - when compared to control group, as determined by ANOVA followed by the Tukey post hoc test.

\section{RESULTS}

The results of our study showed lipid metabolism status in aged rats and also changing in lipid parameters after treatment with fish oil in six weeks treatment.

Our results of biochemical measurements showed changes in concentrations of CHOL, HDL, LDL, nonHDL and its ratios showing cardiovascular and atherosclerosis risk (Table 1).

CHOL concentration, LDL concentration and non HDL concentrations significantly decreased in treated group at the end when compared to treated group at start

Table 3. Plasma phospholipids fatty acid profile in aged Wistar rats

\begin{tabular}{lcc}
\hline Fatty acids (\%) & \multicolumn{1}{c}{ Before } & After (Fish oil) \\
\hline $16: 0$ & $28.34 \pm 1.89$ & $26.23 \pm 3.75$ \\
$16: 1$ & $0.73 \pm 0.50$ & $0.77 \pm 0.42$ \\
$18: 0$ & $24.36 \pm 1.46$ & $27.91 \pm 2.98$ \\
$18: 1, n-9$ & $8.23 \pm 3.03$ & $6.89 \pm 0.91$ \\
$18: 1, n-7$ & $2.04 \pm 0.60$ & $2.90 \pm 0.50$ \\
$18: 2$ & $14.07 \pm 1.41$ & $17.23 \pm 1.66^{* *}$ \\
$20: 3$ & $0.69 \pm 0.13$ & $1.31 \pm 0.46^{*}$ \\
$20: 4$ & $14.77 \pm 2.43$ & $8.87 \pm 2.21^{*}$ \\
$20: 5$ & $0.41 \pm 0.22$ & $0.72 \pm 0.21^{*}$ \\
$22: 4$ & $0.36 \pm 0.19$ & $0.47 \pm 0.23$ \\
$22: 5$ & $0.67 \pm 0.17$ & $1.54 \pm 0.40^{* *}$ \\
$22: 6$ & $5.34 \pm 1.39$ & $5.03 \pm 1.19$ \\
SFA & $52.70 \pm 1.18$ & $54.13 \pm 3.97$ \\
MUFA & $10.99 \pm 3.94$ & $10.56 \pm 0.88$ \\
PUFA & $36.32 \pm 3.65$ & $35.18 \pm 4.12$ \\
$n-3$ & $6.42 \pm 1.46$ & $7.28 \pm 1.61$ \\
$n-6$ & $29.89 \pm 3.02$ & $27.89 \pm 3.10$ \\
\hline
\end{tabular}

$* p<0.05 ; * * p<0.01-$ student test. and also vs. control group. Concentration of LDL fraction significantly decreased in treated group at the end as compared to treated group at start and also vs. control group. Concentration of TG was slightly decreased at the end when compared to start of the study as well as $v s$. control group, although the changes were not statistically significant (Table1). The ratios between CHOL and HDL, (as a factor of cardiovascular risk $^{28}$ ), were significantly lower in treated group at the end than in the same group at start and also than in control group. Furthermore, LDL/HDL ratios (as a factor of atherosclerosis risk $^{28}$ ), were significantly decreased after treatment when compared to baseline values and to control group as well (Table 2).

Concentrations of plasma and liver phospholipids profiles were also changed after supplementation with fish oil capsules in six weeks treatment. Our results showed statistically significant increasing in plasma phospholipids concentrations of LA, dihomo- $\gamma$ linolenic acid, EPA and DPA and decreasing in AA concentration after supplementation with fish oil (Table 3).

Plasma phospholipids concentrations of SFA, MUFA, PUFA, $n-3, n-6$ showed no statistically significant changes (Table 3 ), while liver phospholipids concentrations of SFA, MUFA, PUFA, $n-3$ increased and $n-6$ and $n-6 / n-3$ ratio decreased after supplementation of fish oil when compared to control group (Table 4). In addition, liver phospholipids FAs results demonstrated significantly increased concentrations of vascenic acid, LA, EPA, and DPA and significantly reduced concentration of AA after supplementation with fish oil, than in control group (Table 4).

Treatment with fish oil also induced changes in estimated activity of desaturase and elongase system in both plasma and liver phosholipids. The estimated activities of $\Delta 4, \Delta 5, \Delta 6$ desaturase in plasma decreased after the teatment, while the estimated activities of $\Delta 4$ 
Table 4. Liver phospholipids fatty acid profile in aged Wistar rats

\begin{tabular}{lcc}
\hline Fatty acids (\%) & control group & $\omega-3$ group \\
\hline $16: 0$ & $18.15 \pm 1.18$ & $25.50 \pm 2.39^{* * *}$ \\
$16: 1$ & $0.65 \pm 0.32$ & $0.69 \pm 0.12$ \\
$18: 0$ & $26.38 \pm 1.88$ & $26.27 \pm 3.03$ \\
$18: 1, n-9$ & $5.38 \pm 1.12$ & $4.75 \pm 0.49$ \\
$18: 1, n-7$ & $2.03 \pm 0.34$ & $2.95 \pm 0.63^{*}$ \\
$18: 2$ & $14.43 \pm 1.37$ & $17.35 \pm 1.68^{* *}$ \\
$18: 03$ & $0.05 \pm 0.01$ & $0.07 \pm 0.01$ \\
$20: 3$ & $1.68 \pm 0.36$ & $1.73 \pm 0.54$ \\
$20: 4$ & $23.02 \pm 3.26$ & $10.99 \pm 1.42^{* * *}$ \\
$20: 5$ & $0.50 \pm 0.09$ & $2.64 \pm 0.67 * * *$ \\
$22: 4$ & $0.22 \pm 0.11$ & $0.53 \pm 0.44$ \\
$22: 5$ & $0.98 \pm 0.11$ & $1.52 \pm 0.08^{* * *}$ \\
$22: 6$ & $4.90 \pm 1.04$ & $5.00 \pm 0.24$ \\
SFA & $51.77 \pm 2.72$ & $44.54 \pm 1.033^{* * *}$ \\
MUFA & $8.4 \pm 0.97$ & $8.05 \pm 1.09^{* * *}$ \\
PUFA & $28.85 \pm 1.82$ & $22.76 \pm 1.06^{* * *}$ \\
$n-3$ & $9.23 \pm 0.84$ & $6.44 \pm 1.10^{* * *}$ \\
$n-6$ & $30.61 \pm 2.64$ & $39.35 \pm 3.73^{* * *}$ \\
$n-6 / n-3$ & $3.35 \pm 0.51$ & $6.30 \pm 1.46^{* * *}$ \\
\hline$* p<0.05 ; * * p<0.01 ; * * *<0.001$ & - student test.
\end{tabular}

and $\Delta 5$ desaturases were also decreased in liver. The estimated activity of elongase in liver was also decreased comparing to control group (Tables 5 and 6).

\section{DISCUSSION}

Membrane fluidity depends on alterations in the phospholipid fatty acid composition, e.g. on FAs chain length, degree and type of unsaturation. The saturated FAs (16:0 and 18:0), as well as the ratio between saturated and unsaturated FAs tend to decrease membrane lipid fluidity. ${ }^{29,30}$ Dietary FAs profile influences tissue FAs composition as well as membrane FAs composition and membrane protein function. ${ }^{31}$ Feeding animals diets with a high ratio of PUFAs improved insulin action, glucose transport and other cellular processes. ${ }^{32}$

Our results are in line with the other studies. In a study by Calviello et al. ${ }^{33}$ Wistar rats received by gavage EPA or DHA (360 mg/kg/day), for 1 to 4 weeks. EPA treatment increased EPA and DPA content in plasma. DHA treatment mainly increased DHA content. Both tretment decreased AA content and $n-6 / n-3$ PUFA ratio in the membranes, withouht modifying the unsaturation index. ${ }^{33}$

Froyland et al. ${ }^{34}$ clarified the effects of purified esters of EPA and DHA on lipid metabolism in rats. The animals were fed by orogastric intubation 1 $\mathrm{g} / \mathrm{kg} /$ day for 3 months. That study showed that esters of
Table 5. Estimated liver desaturase and elongase activities in control and treated group in aged Wistar rats

\begin{tabular}{lcc}
\hline & control group & after treatment \\
\cline { 2 - 3 } $22: 6 / 22: 5(\Delta 4)$ & $5.07 \pm 1.26$ & $3.29 \pm 0.25^{\# \#}$ \\
20:4/20:3 $(\Delta 5)$ & $14.37 \pm 4.05$ & $6.72 \pm 1.59^{\# \#}$ \\
20:3/18:2 $(\Delta 6)$ & $0.12 \pm 0.03$ & $0.099 \pm 0.02$ \\
18:1/18:0 $(\Delta 9)$ & $0.20 \pm 0.03$ & $0.18 \pm 0.04$ \\
18:0/16:0 elongase & $1.46 \pm 0.18$ & $1.04 \pm 0.17^{\# \# \#}$ \\
\hline$\# p<0.01 ;{ }^{\# \#} p<0.001-$ student test. &
\end{tabular}

EPA (EPA-EE) and esters of DHA (DHA-EE), lowered plasma cholesterol level, whereas only EPA-EE lowered the amount of plasma triacylglycerol. In liver microsomes EPA-EE raised HMG-CoA reductase and acyl-CoA cholesterol acyltransferase activities. An inhibition of HMG-CoA reductase activity in DHA-EE treated rats maight have contributed to the hypocholesterolemic effect. ${ }^{34}$

According to Leigh-Firbank et al. ${ }^{35}$ fish oil can reduce TG and cardiovascular risk. Increases in platelet EPA and DHA were postively associated with the increase in LDL oxidation. Animal model ${ }^{36}$ and in vitro results $^{37}$ suggested that EPA rather than DHA may be a reducing TG agent. $n-3$ PUFAs supplementation showed an impact on overall LDL concentraton with 5 to $10 \%$ increase. ${ }^{36}$ This study indicated that despite an increase in LDL oxidation and LDL concentration following fish oil intervention there was an increase in LDL density which may have counterected these proatherogenic trends.

Comparing our results on plasma phospholipids FAs profile in aged rats (control group) in our study, with Wistar rats of 8 weeks of age, there were no significant changes ${ }^{38}$ in SFA, MUFA and PUFA concentrations, as well as in separate FAs percentage. Diet treatment changes in profile of $\mathrm{FAs}^{39}$ depend on type of diet, suggesting that amount of fat has a greater influence on obesity and FAs profile than the type of fat consumed. ${ }^{34}$

Plasma FAs can enter liver when unesterified by passive diffusion or facilitated transpor $\mathrm{t}^{40}$ or via hepatocyte lipoprotein receptors when esterified within lipoproteins. ${ }^{41}$ Comparing FAs composition of total liver

Table 6. Estimated plasma desaturase and elongase before and after treatment in aged Wistar rats

\begin{tabular}{lcc}
\hline & before treatment & after treatment \\
\cline { 2 - 3 } $22: 6 / 22: 5(\Delta 4)$ & $8.48 \pm 3.37$ & $3.32 \pm 0.56^{* *}$ \\
20:4/20:3 $(\Delta 5)$ & $21.57 \pm 4.01$ & $7.48 \pm 3.56^{* * *}$ \\
20:3/18:2 $(\Delta 6)$ & $0.05 \pm 0.01$ & $0.07 \pm 0.02^{*}$ \\
18:1/18:0 $(\Delta 9)$ & $0.34 \pm 0.14$ & $0.25 \pm 0.05$ \\
18:0/16:0 elongase & $0.86 \pm 0.10$ & $1.09 \pm 0.22$ \\
\hline
\end{tabular}

$* p<0.01 ; * * p<0.01 ; * * * p<0.001-$ student test. 
phospholipids of aged Wistar rats in our study, with 8 weeks old rats from the Ristic-Medic et al., ${ }^{42}$ we found that overall SFA were significantly increased, MUFA and PUFA significantly decreased in our aged Wistar rats. Treatment with fish oil capsules in our experiment improved overall unsaturation, increasing $n-3$, but decreasing $n-6$ FAs. When compared our results with Wistar rats aged 8 weeks ${ }^{42}$ this unsaturation index was even lower, suggesting that old rats had better response to fish oil therapy.

Comparing FAs composition of total liver phospholipids of aged Wistar rats in our study with FAs composition of total heart phospholipids of 6 to 8 weeks old Wistar rats, ${ }^{43}$ SFA were higher and MUFA and PUFA were lower in older rats. Also, oleic acid and LA were both significantly lower, while AA was higher in liver of aged rats. It seems that aging itself is a risk factor and at least in part lead to higher saturation of FAs in plasma and tissues phospholipids as well.

Furthermore, we compared our results of liver FA composition with 3 weeks Wistar rats in a study by Kawai et al. ${ }^{44}$ The baseline percentage of total SFA was higher in our aged rats, but with after fish oil supplementation, unsaturated index increased more than in 3 weeks Wistar rats used in that study. ${ }^{44}$

Dietary FAs composition is significantly correlated to cell membrane FAs composition. $n-3$ FAs of fish oils are known to be elongated and are desaturated to a greated extent than $n-6 \mathrm{FAs}$, producing a higher unsaturation index in fish oil-fed rats. ${ }^{45}$ Carpentier et $a l .^{46}$ support the view that the FAs pattern of liver phospholipids and TG can be evaluated from measurment made in plasma for the same lipids. The measurment of selected long-chain $n-3$ PUFAs in erythrocytes could help to identify those subjects with a relative deficiency in those $n-3$ FAs and to assess the correction of such a defect in response to an increase in their dietary intake. $^{46}$

Many studies have examined the regulation of mammals elongases at the level of enzyme activity. Also, examinations are focused on nutritional and tissue-specific regulation of elonase expression more recently. Elongases and desaturases work in concert to synthesize MUFAs and PUFAs. In rat liver Elovl-5 is predominant elongase. ${ }^{47}$ Rat hepatic Elovl-5 and $\Delta 5$ and $\Delta 6$ desaturases are coordinately regulated by diet and during postnatal development. The low elongase activity in fetal livers correlates with the Elovl-1, Elovl-2 and Elovl-6 low expression in both fetal and adults liver. Elovl-5 is responsible for major FAs elongation and is capable of elongating 16:0, 18:0, 20:0, 22:0 and 24:0 saturated FAs rather then monounsaturated $(18: 1, n-9)$ and polyunsaturated $(20: 4, n-6)$ FAs.

Rat hepatic Elovl-5 expression is regulated at the pretranslational level by dietary $n-3$ PUFAs and PPAR $\alpha$ agonist and during postnatal development. Unlike $\Delta 5$, $\Delta 6$ and $\Delta 9$ desaturases, the elongases do not display uniform responce to fish oil or PPAR $\alpha$ agonist. ${ }^{48}$ For example; rat heart does not synthesize DHA from $\alpha$-LNA owing to the absence of elongase-2, but must obtain its DHA entirely from plasma.

In this paper we were not measure the activities of desaturase and elongase system, but we estimated their activities according to ratios of specifics FAs. However, we found a significant decrease in estimated activities of $\Delta 4$ and $\Delta 5$ in both plasma and liver of treated rats. In addition, a slight but significant increase in estimated $\Delta 6$ desaturase activity was shown in liver, while a decrease in the estimated activity of elongase was detected in plasma of Wistar rats after fish oil supplementation.

\section{CONCLUSION}

The results of our study showed that 6 weeks of fish oil treatment of aged Wistar rats significantly improved lipid status and risk factors for atherosclerosis and cardiovascular disease. Also, we found a significant increase in most favorable FAs of $n-3$ series (EPA, DPA etc.), and decrease of saturated as well as $n-6$ PUFAs, particularly AA, in both plasma and liver, indicating markedly improved FAs profile of phospholipids in supplemented rats. The treatment with fish oil affected the estimated activities of desaturase and elongase system as well. Considering changes in FAs profile in phospholipids during aging, fish oil supplementation could have beneficial effects, and our future examination will address it.

Acknowledgements. This work was supported by the Project III 41030 financed by the Ministry of Science Republic of Serbia. Part of this paper was presented at the $10^{\text {th }}$ Congress of the Croatian Society of Biochemistry and Molecular Biology with international participation in Opatija, Croatia $\left(15^{\text {th }}\right.$ to $18^{\text {th }}$, September, 2010).

\section{REFERENCES}

1. A. P. Simopoulos, Am. J. Clin. Nutr. 55 (1992) 760-761.

2. M. Bretscher, Sci. Am. 253 (1985) 100-108.

3. N. Unwin and R. Henderson, Sci. Am. 250 (1984) 978-94.

4. J. Storch and A. M. Kleinfeld, Trends Biochem. Sci. 10 (1985) 418-421.

5. P. Avogaro, G. Bitoldo, and G. Gazzolatao, Drugs affecting lipid metabolism, Springer-Verlag Berlin, Heidelberg, 1987, 407-409.

6. W. S. Harris, J. V. Pottala, S. A. Sands, and P. G. Jones, Am. J. Clin. Nutr. 86 (2007) 1621-1625.

7. M. B. Katan, J. P. Deslypere, A. P. van Birgelen, M. Penders, and M. Zegwaard, J. Lipid. Res. 38 (1997) 2012-2022.

8. V. Mougios, C. Kotzamanidis, C. Koutsari, and S. Atsopardis, Metabolism 44 (1995) 681-688.

9. P. C. Calder, Braz. J. Med. Biol. Res. 36 (2003) 433-446.

10. T. Popović, M. Ranić, P. Bulajić, M. Miličević, A. Arsić, V. Vučić, and M. Glibetić, J. Clin. Biochem. Nutr. 45 (2009) 370-375. 
11. C. Chapman, L. Morgan, and M. Murphy, J. Nutr. 130 (2000) 146-151.

12. S. D. Clarke, M. Turini, and D. Jump, Prostagl. Leukotr. ESS. 57 (1997) 65-69.

13. B. Daggy, C. Arost, and A. Bensadoun, BBA - Lipids and Lipid Metabolism. 920 (1987) 293-300.

14. G. Barcelo-Coblijn, J. C. Murphy, R. Othman, H. M. Moghadasian, T. Kashour, and K. J. Friel, Am. J. Clin. Nutr. 88 (2008) 801-809.

15. K. S. Layne, Y. K. Goh, J. A. Jumpsen, E. A. Ryan, P. Chow, and M. T. Clandinin, J. Nutr. 126 (1996) 2130-2140.

16. G. K. Paschos, L. S. Rallidis, and G. K. Liakos, Br. J. Nutr. 92 (2004) 649-655.

17. A. Rigoti, M. P. Marzalo, and F. Nervi, Current topics in membranes 40 (1994) 579-615.

18. A. M. Giudetti, S. Sabetta, R. di Summa, M. Leo, F. Damiano, L. Siculella, and V. G. Gnoni, J. Lipid. Res. 44 (2003) 2135-2141.

19. F. Forcheron, S. Cachefo, C. Thevenon, C. Pinteur, and M. Beylot, Diabetes 51 (2002) 3486-3491

20. E. Shrago, T. Spenneta, and E. Gordon, J. Biol. Chem. 244 (1969) 905-912.

21. B. Mitttendorfer and L. Sidossis, Am. J. Clin. Nutr. 73 (2001) 892-899.

22. R. Jeffcoat, Essays Biochem. 15 (1979) 1-36.

23. V. Tepsić, M. Pavlović, D. Ristić Medić, J. Tepsić, M. Miličević, and M. Glibetić, Acta. Vet. Beograd 58 (2008) 33-41.

24. M. F. Lopes-Virella, P. Stone, S. Ellis, and J. A. Colwell, Clin. Chem. 23 (1977) 882-884.

25. W. T. Friedewald, L. I. Levy, and D. S. Fredrickson, Clin. Chem. 18 (1972) 499-502.

26. J. Folch, M. Lees, S. Sloane, and G. H. Stanley, J. Biol. Chem. (1957) 497-509.

27. Z. Cvetković, V. Vučić, B. Cvetković, M. Petrović, D. RistićMedić, J. Tepsić, and M. Glibetić, Ann. Hematol. 89 (2010) $775-782$.

28. D. Ristić Medić, V. Ristić, A. Arsić, M. Postić, G. Ristić, V. Blazenčić Mladenović, and J. Tepsić, Nutr. Metab. Cardiovas. 16 (2006) 395-404.

29. D. Ristić-Medić, G. Ristić, V Tepsić, and G. N. Ristić, J. Nutr.
Sci. Vitaminol. 49 (2003) 367-374.

30. V. Tepsić, V. Ristić, N. Ristić, M. Vasiljević, and M. Pecelj-Gec, Physiol. Res. 47 (1998) 413-418.

31. G. R. Hynes, J. Heshka, K. Chadee, and P. J. Jones, J. Lipid Res. 44 (2003) 893-901.

32. D. A. Pan and L. H. Storlien, J. Nutr. 123 (1993) 512-519.

33. G. Calviello, P. Palozza, P. Franceschelli, and G. M. Bartoli, Lipids. 32 (1997) 1075-1083.

34. L. Froyland, H. Vaagenes, D. K. Asiedu, A. Garras, O. Lie, and R. K. Berge, Lipids 31 (1996) 169-178.

35. E. C. Leigh-Firbank, A. M. Minihane, D. S. Leake, J. W. Wright, M. C. Murphy, B. A. Griffin, and C. M. Wiliams, $B J N 87$ (2002) 435-445

36. R. K. Berge, L. Madsen, H. Vaagenes, K. J. Tronstad, M. Gottlicher, and A. C. Rustan, BJ 343 (1990) 191-197.

37. W. H. Wong, E. A. Fisher, and J. B. March, Ateriosclerosis 9 (1989) 836-841

38. W. S. Harris, J. Lipid Res. 30 (1989) 127-807.

39. V. Tepsić, M. Pavlović, D. Ristić-Medić, V. Ristić, N. Lekić, J. Tepsić, J. Debeljak Martačić, M. Miličević, M. Glibetić, Acta vet Beograd. 58 (2008) 33-41.

40. E. J. Dement, G. V. Richieri, and A. M. Kleinfeld, Biochem. J. 63 (2002) 809-815.

41. Stahl A: Pflugers. Arch. 447 (2004) 722-727.

42. D. Ristić-Medić, G. Ristić, V. Tepsic, G. N. Ristić, J. Nutr. Sci. Vitaminol. 49 (2003) 367-374.

43. V. Tepsić, V. Ristić, N. Ristić, M. Vasiljević, and M. Pecelj-Gec, Physiol. Res. 47 (1998) 413-418.

44. Y. Kawai, H. Fujii, M. Okada, Y. Tsuchie, K. Uchida, and T. Osawa, J. Lipid. Res. 47 (2006) 1387-95.

45. C. Jen, A. Buison, M. Pellizzon, F. Ordiz, L. Santa Ana, and J. Brown, Exp. Biol. Med. 228 (2003) 843-849.

46. Y. Carpetier, L. Portois, A. Sener, and W. Y. Malaisse, Int. J. Mol. Med. 22 (2008) 255-262.

47. Y. Wang, D. Botolin, B. Christian, J. Busik, J. Xu, and D. B. Jump, J. Lipid. Res. 46 (2005) 706-715.

48. M. Igarashi, K. Ma, L. Chang, J. M. Bell, and S. I. Rapoport, $J$ Lipid. Res. 49 (2008) 1735-1745. 